Pacific Journal of Mathematics

DETERMINATIONS OF JACOBSTHAL SUMS 


\title{
DETERMINATIONS OF JACOBSTHAL SUMS
}

\author{
RONALD J. EvanS
}

\begin{abstract}
The sign ambiguities are resolved in evaluations of Jacobsthal sums $\sum_{m=1}^{p}\left(m\left(m^{k}+a\right) / p\right)$ for $k=2,3,4,6,10$, and 12 , where $(/ p)$ denotes the Legendre symbol.
\end{abstract}

1. Introduction. For a positive even integer $e=2 n$, a prime $p=e f$ +1 , and an integer $a$ prime to $p$, define the Jacobsthal sum of order $e$ by

$$
\varphi_{n}(a)=\sum_{m=1}^{p}\left(\frac{m\left(m^{n}+a\right)}{p}\right)
$$

where $(/ p)$ denotes the Legendre symbol. In [1, $\$ 4]$, the values of Jacobsthal sums $\varphi_{n}(a)$ of orders $e=4,6,8,12,20,24$ are given up to some sign ambiguities. The purpose of this paper is to show how the precise values of $\varphi_{n}(a)$ can be found.

In $\S 3$, we give congruence conditions $(\bmod p)$ which determine the correct choices of \pm signs. The computational complexity of these determinations for large $p$ is much less than that of computing $\varphi_{n}(a)$ directly from the definition.

In $\S 4$, we describe a method for determining the correct choices of \pm signs by congruence conditions $(\bmod a)$, when $a$ is prime. If $a$ is small compared with $p$, then the determinations in $\$ 4(\bmod a)$ turn out to be computationally simpler than those in $\$ 3(\bmod p)$.

The cases $e=4,6$ and $e=8$ have already been treated by Hudson and Williams in [2] and [3], respectively. We employ different techniques based on Jacobi sums which work for all values $e=4,6,8,12,20,24$. Each of these values of $e$ is considered in $\$ 3$, but in $\$ 4$, only the case $e=12$ is treated, for brevity.

It will be convenient to introduce the notation $F_{e}(a)$ for the sum

$$
F_{e}(a)=\sum_{m=1}^{p}\left(\frac{m\left(m^{e / 2}-a\right)}{p}\right)=\varphi_{n}(-a) .
$$

An evaluation of $F_{e}(a)$ immediately yields one for $\varphi_{n}(a)$, since $[4,(7)]$

$$
F_{e}(a)=\varphi_{n}(-a)=\varphi_{n}(a)(-1)^{f n+f} .
$$

In the sequel, attention will be focused on $F_{e}(a)$. 
2. Notation and Jacobi sums. For a character $\lambda(\bmod p)$, define the Jacobi sums

$$
J(\lambda)=\sum_{m=1}^{p} \lambda(m) \lambda(1-m), \quad K(\lambda)=\lambda(4) J(\lambda) .
$$

Write $p=e f+1$. For each value of $e=4,6,8,12,20,24$, fix a character $\chi=\chi_{e}(\bmod p)$ of order $e$. Let $P$ be the prime ideal divisor of $p$ in $\mathbf{Z}[\exp (2 \pi i / e)]$ chosen such that

$$
\chi(\alpha) \equiv \alpha^{(p-1) / e}=\alpha^{f} \quad(\bmod P)
$$

for all $\alpha \in \mathbf{Z}[\exp (2 \pi i / e)]$. It is easily seen that

$$
K(\chi) \equiv 0 \quad(\bmod P) .
$$

In $[1, \S 3]$ one finds the following evaluations of Jacobi sums $K(\chi)$ of orders $e=4,6,8,12,20,24$ in terms of parameters in quadratic partitions of $p$.

(4) $K\left(\chi_{4}\right)=a_{4}+i b_{4}, \quad$ where $p=a_{4}^{2}+b_{4}^{2}, a_{4} \equiv-(2 / p) \quad(\bmod 4)$;

$$
\begin{aligned}
\left(\frac{-1}{p}\right) K\left(\chi_{6}\right)=K\left(\chi_{6}^{2}\right) & =a_{3}+i b_{3} \sqrt{3}, \\
\text { where } p & =a_{3}^{2}+3 b_{3}^{2}, a_{3} \equiv-1 \quad(\bmod 3) ;
\end{aligned}
$$

(6) $K\left(\chi_{8}\right)=a_{8}+i b_{8} \sqrt{2}$, where $p=a_{8}^{2}+2 b_{8}^{2}, a_{8} \equiv-1 \quad(\bmod 4)$;

$$
K\left(\chi_{12}\right)= \begin{cases}-a_{4}-i b_{4}, & \text { if } 3 \mid a_{4}, \\ a_{4}+i b_{4}, & \text { if } 3 \nmid a_{4},\end{cases}
$$

where

$$
\begin{gathered}
K\left(\chi_{12}^{3}\right)=a_{4}+i b_{4} \text { as in }(4) ; \\
K\left(\chi_{24}\right)=a_{24}+i b_{24} \sqrt{6}, \quad \text { where } p=a_{24}^{2}+6 b_{24}^{2}, \\
a_{24} \equiv a_{8}(\bmod 3), \quad \text { with } K\left(\chi_{24}^{3}\right)=a_{8}+i b_{8} \sqrt{2} \text { as in }(6) ; \\
K\left(\chi_{20}\right)= \begin{cases}a_{20}+i b_{20} \sqrt{5}, & \text { if } 5 \nmid a_{4}, \\
i a_{20}-b_{20} \sqrt{5}, & \text { if } 5 \mid a_{4},\end{cases}
\end{gathered}
$$

where

$$
p=a_{20}^{2}+5 b_{20}^{2} \text { and } a_{20} \equiv \begin{cases}a_{4}(\bmod 5), & \text { if } 5 \nmid a_{4}, \\ b_{4}(\bmod 5), & \text { if } 5 \mid a_{4},\end{cases}
$$

with $K\left(\chi_{20}^{5}\right)=a_{4}+i b_{4}$ as in (4). 
3. Congruence conditions $(\bmod p)$. This section is to be read in conjunction with $[1, \S 4]$. We consider only those values of $a$ for which the evaluations of $F_{e}(a)$ in $[1, \S 4]$ have sign ambiguities, and we resolve these ambiguities with congruence conditions $(\bmod p)$, for $e=4,6,8,12,20$, 24.

Case 1. $e=4,(a / p)=-1$.

The proof in [1, Theorem 4.4] shows that

$$
F_{4}(a)=2 \operatorname{Re}(\bar{\chi}(a) K(\chi))=-2 b_{4} i \chi(a)= \pm 2 b_{4} .
$$

To determine the correct sign, it remains to find $F_{4}(a)(\bmod p)$. By (3) and $(4),-i b_{4} \equiv a_{4}(\bmod P)$. Thus by $(10)$ and $(2), F_{4}(a) \equiv 2 a_{4} a^{f}(\bmod P)$, so

$$
F_{4}(a) \equiv 2 a_{4} a^{f} \quad(\bmod p)
$$

REMARK. While it takes the computer $O(p)$ operations to compute $F_{4}(a)$ directly from the definition (1), it requires at most $O(\sqrt{p})$ operations to compute $F_{4}(a)$ from $(10)$ and (11), since $a^{f}(\bmod p)$ can be computed in $O(\log p)$ steps.

Case 2. $e=6, a$ is noncubic $(\bmod p)$.

Write $\lambda=\chi_{6}^{2}$. Note that $\lambda(a)=(-1 \pm i \sqrt{3}) / 2$. The proof in [1, Theorem 4.2] shows that

$$
\begin{aligned}
F_{6}(a) & =-1+2 \operatorname{Re}(\bar{\lambda}(a) K(\lambda)) \\
& =-1-a_{3}+2 b_{3} \sqrt{3} \operatorname{Im} \lambda(a)=-1-a_{3} \pm 3 b_{3} .
\end{aligned}
$$

It remains to determine $F_{6}(a)(\bmod p)$. By (3) and (5), $a_{3} \equiv-i b_{3} \sqrt{3}$ $(\bmod P)$, so by $(12)$ and $(2)$,

$$
F_{6}(a) \equiv a_{3}\left(a^{2 f}-a^{4 f}\right)-1-a_{3} \equiv 2 a_{3} a^{2 f}-1 \quad(\bmod p) .
$$

Case 3. $e=8,(a / p)=-1$.

From the proof in [1, Theorem 4.6],

$$
\begin{aligned}
F_{8}(a) & =-2 \operatorname{Re}\left(K(\chi)\left(\chi(a)+\chi^{3}(a)\right)\right) \\
& =-2 i b_{8} \sqrt{2}\left(\chi(a)+\chi^{3}(a)\right)= \pm 4 b_{8} .
\end{aligned}
$$

Thus,

$$
F_{8}(a) \equiv 2 a_{8}\left(a^{f}+a^{3 f}\right) \quad(\bmod p)
$$


Case 4. $e=12,(a / p)=-1$.

Subcase 4A. $3 \mid a_{4}, a$ is cubic $(\bmod p)$.

By $[1,(4.3)]$,

$$
F_{12}(a)=6 \operatorname{Re}\left(\chi(a)\left(a_{4}+i b_{4}\right)\right)=6 \chi(a) i b_{4}= \pm 6 b_{4} .
$$

By (3) and (7), $a_{4} \equiv-i b_{4}(\bmod P)$, so

$$
F_{12}(a) \equiv-6 a_{4} a^{f} \quad(\bmod p) .
$$

Subcase 4B. $3+a_{4}$.

By $[\mathbf{1},(4.5)]$,

$$
\begin{aligned}
& F_{12}(a)=2 b_{4} / \operatorname{Im} \chi(a) \\
& =4 i b_{4} /\left(\chi(a)+\chi^{5}(a)\right)= \begin{cases} \pm 4 b_{4}, & \text { if } a \text { is noncubic }(\bmod p) \\
\pm 2 b_{4}, & \text { if } a \text { is cubic }(\bmod p) .\end{cases}
\end{aligned}
$$

Thus,

$$
F_{12}(a) \equiv-4 a_{4} /\left(a^{f}+a^{5 f}\right) \quad(\bmod p)
$$

Case 5. $e=24,(a / p)=-1$.

This case is slightly different than those above in that two congruence conditions are required to determine $F_{24}(a)$. From the proof in [1, Theorem 4.10],

$$
F_{24}(a)=A_{24}+B_{24},
$$

where

$$
\begin{aligned}
A_{24} & =-2 \operatorname{Re}\left(\left(a_{8}+i b_{8} \sqrt{2}\right)\left(\chi^{3}(a)+\chi^{9}(a)\right)\right) \\
& =-2 i b_{8} \sqrt{2}\left(\chi^{3}(a)+\chi^{9}(a)\right)= \pm 4 b_{8}
\end{aligned}
$$

and

$$
\begin{aligned}
B_{24} & =-2 \operatorname{Re}\left(\left(a_{24}+i b_{24} \sqrt{6}\right)\left(\chi(a)+\chi^{5}(a)+\chi^{7}(a)+\chi^{11}(a)\right)\right) \\
& =-2 i b_{24} \sqrt{6}\left(\chi(a)+\chi^{5}(a)+\chi^{7}(a)+\chi^{11}(a)\right) \\
& = \begin{cases} \pm 12 b_{24}, & \text { if } a \text { is noncubic }(\bmod p) \\
0, & \text { if } a \text { is cubic }(\bmod p) .\end{cases}
\end{aligned}
$$

It remains to determine $A_{24}$ and $B_{24}(\bmod p)$. Since $a_{8} \equiv-i b_{8} \sqrt{2}$ and $a_{24} \equiv-i b_{24} \sqrt{6}(\bmod P)$, we have

$$
A_{24} \equiv 2 a_{8}\left(a^{3 f}+a^{9 f}\right) \quad(\bmod p)
$$


and

$$
B_{24} \equiv 2 a_{24}\left(a^{f}+a^{5 f}+a^{7 f}+a^{11 f}\right) \quad(\bmod p) .
$$

Case 6. $e=20$.

This case is similar to Case 5, so we omit some details. From the proof in [1, Theorem 4.13],

$$
F_{20}(a)=A_{20}+B_{20},
$$

where

$$
A_{20}=2 \operatorname{Re}\left\{\chi^{5}(a)\left(a_{4}-i b_{4}\right)\right\}
$$

and

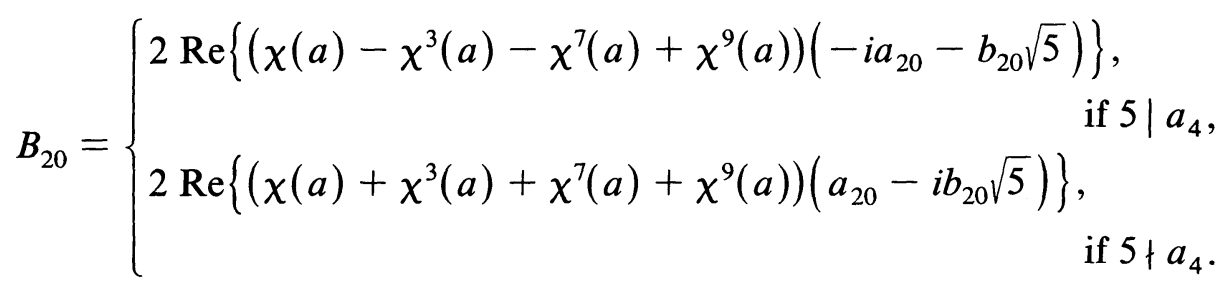

It remains to determine $A_{20}$ and $B_{20}$ in each of the subcases below.

Subcase 6A. $5 \mid a_{4},(a / p)=1$, a nonquintic $(\bmod p)$.

Here $A_{20}= \pm 2 a_{4}$ and $B_{20}= \pm 10 b_{20}$, with

$$
A_{20} \equiv 2 a_{4} a^{5 f}(\bmod p)
$$

- and

$$
B_{20} \equiv 2\left(a^{f}-a^{3 f}-a^{7 f}+a^{9 f}\right) a_{4} a_{20} / b_{4} \quad(\bmod p) .
$$

Observe that there is no sign ambiguity in the right member of (17), since $a_{20} / b_{4} \equiv 1(\bmod 5)$, as is noted after $(9)$.

Subcase 6B. 5| $a_{4},(a / p)=-1$.

Here,

$$
A_{20}= \pm 2 b_{4} \quad \text { and } \quad B_{20}= \begin{cases} \pm 8 a_{20}, & \text { if } a \text { is quintic }(\bmod p) \\ \pm 2 a_{20}, & \text { if } a \text { is nonquintic }(\bmod p)\end{cases}
$$

with the congruences (16) and (17) again holding. 
Subcase 6C. $5+a_{4},(a / p)=-1$.

Here

$$
A_{20}= \pm 2 b_{4} \quad \text { and } \quad B_{20}= \begin{cases} \pm 10 b_{20}, & \text { if } a \text { is nonquintic }(\bmod p) \\ 0, & \text { if } a \text { is quintic }(\bmod p),\end{cases}
$$

with (16) holding and

$$
B_{20} \equiv 2 a_{20}\left(a^{f}+a^{3 f}+a^{7 f}+a^{9 f}\right) \quad(\bmod p) .
$$

4. Congruence conditions $(\bmod a)$. Throughout this section, $e=12$, $p=12 f+1, \chi$ is a character $(\bmod p)$ of order $12,(a / p)=-1$, and $a$ is prime. From (14) and (15),

$$
F_{12}(a)=t \operatorname{Im} K\left(\chi^{3}\right) / \operatorname{Im} \chi(a)=t b_{4} / \operatorname{Im} \chi(a)= \pm h b_{4}
$$

where

$$
K\left(\chi^{3}\right)=a_{4}+i b_{4}
$$

and

$$
\begin{array}{ll}
h=t=-6, & \text { if } 3 \mid a_{4} \text { and } a \text { is cubic }(\bmod p), \\
h=t=2, & \text { if } 3 \nmid a_{4} \text { and } a \text { is cubic }(\bmod p), \\
h=4, t=2, & \text { if } 3 \nmid a_{4} \text { and } a \text { is noncubic }(\bmod p) .
\end{array}
$$

If the prime $a$ is odd, then $a \nmid b_{4}$, otherwise we would have

$$
p=a_{4}^{2}+b_{4}^{2} \equiv a_{4}^{2} \quad(\bmod a),
$$

which contradicts $(a / p)=-1$. Thus we can resolve the ambiguity in (18) by determining $F_{12}(a)(\bmod a)$, if $a>3$. (Note $a \neq 3$, as $(a / p)=-1$.) For $a=2$, we will resolve the ambiguity by determining $F_{12}(2)$ modulo an appropriate power of 2, in (20) and (21) below.

Case 1. $a=2$.

It is classical [4, p. 107] that

$$
b_{4} \equiv-2 i \chi^{3}(2) \quad(\bmod 8) \text {. }
$$

If 2 is a cubic residue $(\bmod p)$, then

$$
\frac{b_{4}}{\operatorname{Im} \chi(2)}=\frac{i b_{4}}{\chi(2)} \equiv \frac{2 \chi^{3}(2)}{\chi(2)}=-2 \quad(\bmod 8),
$$

so by (18),

$$
F_{12}(2) \equiv-2 t \equiv-4(\bmod 16), \quad \text { if } 2 \text { is cubic }(\bmod p) .
$$


If $3 \nmid a_{4}$ and 2 is noncubic $(\bmod p)$, then

$$
\begin{aligned}
F_{12}(2) & =\frac{2 b_{4}}{\operatorname{Im} \chi(2)}=\frac{4 i b_{4}}{\chi(2)-\bar{\chi}(2)} \equiv \frac{8 \chi^{3}(2)}{\chi(2)-\bar{\chi}(2)} \\
& =\frac{8}{\chi^{10}(2)-\chi^{8}(2)}(\bmod 32) .
\end{aligned}
$$

Since $\chi^{8}(2)=(-1 \pm i \sqrt{3}) / 2$ and $\chi^{10}(2)=(1 \pm i \sqrt{3}) / 2$,

(21) $\quad F_{12}(2) \equiv 8(\bmod 32), \quad$ if $3 \nmid a_{4}$ and 2 is noncubic $(\bmod p)$.

Case 2. $a$ is a prime $>3$.

To determine $F_{12}(a)(\bmod a)$, it suffices, by $(18)$, to determine

$$
S(\chi)=\operatorname{Im} \chi(a) / b_{4}
$$

modulo $a$. To do this, we need some formulas for Gauss sums $G(\psi)$, defined for characters $\psi(\bmod p)$ by

$$
G(\psi)=\sum_{n=1}^{p} \psi(n) \exp (2 \pi i n / p) .
$$

From [1, Theorems 2.2 and 3.1],

$$
G(\chi)^{12}=p J^{4}\left(\chi^{4}\right) K^{6}(\chi)
$$

so by [1, Theorem 3.19],

$$
G(\chi)^{12}=p J^{4}\left(\chi^{4}\right) K^{6}\left(\chi^{3}\right) .
$$

From [1, (3.28) and Theorems 2.2 and 3.1],

$$
G^{5}(\chi) / G\left(\chi^{5}\right)=J^{2}\left(\chi^{4}\right) K^{2}(\chi),
$$

so by [1, Theorem 3.19],

$$
G^{5}(\chi) / G\left(\chi^{5}\right)=J^{2}\left(\chi^{4}\right) K^{2}\left(\chi^{3}\right) .
$$

Here, as in [1, Theorem 3.4],

(24) $2 J\left(\chi^{4}\right)=r_{3}+3 i t_{3} \sqrt{3}, \quad$ where $4 p=r_{3}^{2}+27 t_{3}^{2}, r_{3} \equiv 1(\bmod 3)$.

It is clear from the definition of $G(\chi)$ that, in the ring of algebraic integers,

$$
G^{a}(\chi) \equiv \bar{\chi}^{a}(a) G\left(\chi^{a}\right) \quad(\bmod a) .
$$

We will complete the proof by determining $S(\chi)(\bmod a)$ in $(27)-(30)$ in terms of the parameters $p, r_{3}$, and $a_{4}$ unambiguously defined in (4) and (24). 
Subcase 2A. $a \equiv 5(\bmod 12)$.

By (25) and (23),

$$
\chi^{7}(a) \equiv G^{a-5}(\chi) G^{5}(\chi) / G\left(\chi^{5}\right)=G^{a-5}(\chi) J^{2}\left(\chi^{4}\right) K^{2}\left(\chi^{3}\right) \quad(\bmod a) .
$$

Thus, by (22),

$$
\chi^{7}(a) \equiv p^{(a-5) / 12} J^{(a+1) / 3}\left(\chi^{4}\right) K^{(a-1) / 2}\left(\chi^{3}\right) \quad(\bmod a) .
$$

Replacing $\chi$ by $\chi^{7}$, we obtain

$$
\chi(a) \equiv p^{(a-5) / 12} J^{(a+1) / 3}\left(\chi^{4}\right) K^{(a-1) / 2}\left(\bar{\chi}^{3}\right) \quad(\bmod a) .
$$

Each member of (26) is a rational linear combination of $1, i, \sqrt{3}, i \sqrt{3}$ by (19) and (24). The respective coefficients of $i$ must be congruent $(\bmod a)$. Since $\operatorname{Im} \chi(a)$ is rational, it follows that

$$
\operatorname{Im} \chi(a) \equiv-p^{(a-5) / 12} \operatorname{Re} J^{(a+1) / 3}\left(\chi^{4}\right) \operatorname{Im} K^{(a-1) / 2}\left(\chi^{3}\right) \quad(\bmod a)
$$

so

(27) $S(\chi) \equiv-p^{(a-5) / 12} b_{4}^{-1} \operatorname{Re} J^{(a+1) / 3}\left(\chi^{4}\right) \operatorname{Im} K^{(a-1) / 2}\left(\chi^{3}\right)(\bmod a)$.

For example, when $a=5$, (27) yields

$$
\begin{aligned}
S(\chi) & \equiv\left(-4 b_{4}\right)^{-1} \operatorname{Re}\left(r_{3}+3 i t_{3} \sqrt{3}\right)^{2} \operatorname{Im}\left(a_{4}+i b_{4}\right)^{2} \\
& \equiv 2 a_{4}\left(r_{3}^{2}-27 t_{3}^{2}\right) \quad(\bmod 5) .
\end{aligned}
$$

Subcase 2B. $a \equiv 7(\bmod 12)$.

By (25) and (23),

$$
\begin{aligned}
\chi^{5}(a) & \equiv G^{a+5}(\chi) \chi(-1) p^{-1} G\left(\chi^{5}\right) / G^{5}(\chi) \\
& \equiv G^{a+5}(\chi) \chi(-1) p^{-1} /\left(J^{2}\left(\chi^{4}\right) K^{2}\left(\chi^{3}\right)\right) \quad(\bmod a) .
\end{aligned}
$$

Thus, by (22),

$$
\chi^{5}(a) \equiv p^{(a-7) / 12} \chi(-1) J^{(a-1) / 3}\left(\chi^{4}\right) K^{(a+1) / 2}\left(\chi^{3}\right) \quad(\bmod a) .
$$

Replacing $\chi$ by $\chi^{5}$, we obtain

$$
\chi(a) \equiv p^{(a-7) / 12}(-1)^{f} J^{(a-1) / 3}\left(\bar{\chi}^{4}\right) K^{(a+1) / 2}\left(\chi^{3}\right) \quad(\bmod a),
$$

so

$$
\begin{aligned}
S(\chi) \equiv & p^{(a-7) / 12}(-1)^{f} \operatorname{Re} J^{(a-1) / 3}\left(\chi^{4}\right) \\
& \times \operatorname{Im} K^{(a+1) / 2}\left(\chi^{3}\right) / b_{4} \quad(\bmod a) .
\end{aligned}
$$


For example, when $a=7$, (28) yields

$$
\begin{aligned}
S(\chi) & \equiv(-1)^{f}\left(4 b_{4}\right)^{-1} \operatorname{Re}\left(r_{3}+3 i t_{3} \sqrt{3}\right)^{2} \operatorname{Im}\left(a_{4}+i b_{4}\right)^{4} \\
& \equiv(-1)^{f} a_{4}\left(r_{3}^{2}-27 t_{3}^{2}\right)\left(2 a_{4}^{2}-p\right) \quad(\bmod 7) .
\end{aligned}
$$

Subcase 2C. $a \equiv 11(\bmod 12)$.

By (25) and (22),

$$
\begin{aligned}
\chi(a) & \equiv p^{-1} \chi(-1) G^{a+1}(\chi) \\
& \equiv p^{(a-11) / 12} \chi(-1) J^{(a+1) / 3}\left(\chi^{4}\right) K^{(a+1) / 2}\left(\chi^{3}\right) \quad(\bmod a)
\end{aligned}
$$

Thus,

$$
\begin{aligned}
S(\chi) \equiv & p^{(a-11) / 12}(-1)^{f} \operatorname{Re} J^{(a+1) / 3}\left(\chi^{4}\right) \\
& \times \operatorname{Im} K^{(a+1) / 2}\left(\chi^{3}\right) / b_{4} \quad(\bmod a) .
\end{aligned}
$$

For example, when $a=11,(29)$ yields

$$
\begin{aligned}
S(\chi) & =(-1)^{f}\left(16 b_{4}\right)^{-1} \operatorname{Re}\left(r_{3}+3 i t_{3} \sqrt{3}\right)^{4} \operatorname{Im}\left(a_{4}+i b_{4}\right)^{6} \\
& \equiv(-1)^{f} a_{4}\left(3 b_{4}^{4}-10 a_{4}^{2} b_{4}^{2}+3 a_{4}^{4}\right)\left(r_{3}^{4}-162 r_{3}^{2} t_{3}^{2}+729 t_{3}^{4}\right) / 8 \\
& \equiv 7 a_{4}(-1)^{f}\left(3 b_{4}^{4}+a_{4}^{2} b_{4}^{2}+3 a_{4}^{4}\right)\left(r_{3}^{4}+3 r_{3}^{2} t_{3}^{2}+3 t_{3}^{4}\right) \quad(\bmod 11) .
\end{aligned}
$$

Subcase 2D. $a \equiv 1(\bmod 12)$.

By (25) and (22),

$$
\chi(a) \equiv G^{a-1}(\bar{\chi}) \equiv p^{(a-1) / 12} J^{(a-1) / 3}\left(\bar{\chi}^{4}\right) K^{(a-1) / 2}\left(\bar{\chi}^{3}\right) \quad(\bmod a) .
$$

Thus,

(30) $S(\chi) \equiv-p^{(a-1) / 12} \operatorname{Re} J^{(a-1) / 3}\left(\chi^{4}\right) \operatorname{Im} K^{(a-1) / 2}\left(\chi^{3}\right) / b_{4} \quad(\bmod a)$.

For example, when $a=13,(30)$ yields

$$
\begin{aligned}
S(\chi) & \equiv-p\left(16 b_{4}\right)^{-1} \operatorname{Re}\left(r_{3}+3 i t_{3} \sqrt{3}\right)^{4} \operatorname{Im}\left(a_{4}+i b_{4}\right)^{6} \\
& \equiv-p a_{4}\left(3 b_{4}^{4}-10 a_{4}^{2} b_{4}^{2}+3 a_{4}^{4}\right)\left(r_{3}^{4}-162 r_{3}^{2} t_{3}^{2}+729 t_{3}^{4}\right) / 8 \\
& \equiv-2 p a_{4}\left(b_{4}^{4}+a_{4}^{2} b_{4}^{2}+a_{4}^{4}\right)\left(r_{3}^{4}+7 r_{3}^{2} t_{3}^{2}+t_{3}^{4}\right) \quad(\bmod 13)
\end{aligned}
$$


Numerical examples.

\begin{tabular}{r|rrrrrrrrrrrr}
$a$ & 5 & 5 & 5 & 7 & 7 & 7 & 11 & 11 & 11 & 13 & 13 & 13 \\
\hline$p$ & 13 & 37 & 157 & 61 & 73 & 157 & 61 & 193 & 337 & 37 & 193 & 229 \\
\hline$F_{12}(a)$ & 12 & 24 & -24 & -24 & 48 & -12 & -12 & 24 & -96 & 24 & -24 & 12
\end{tabular}

\section{REFERENCES}

[1] B. C. Berndt and R. J. Evans, Sums of Gauss, Jacobi, and Jacobsthal, J. Number Theory, 11 (1979), 349-398.

[2] R. H. Hudson and K. S. Williams, Resolution of ambiguities in the evaluation of cubic and quartic Jacobsthal sums, Pacific J. Math., 99 (1982), 379-386.

[3] An application of a formula of Western to the evaluation of certain Jacobsthal sums, Acta Arith., (to appear).

[4] E. Lehmer, On the number of solutions of $u^{k}+D \equiv w^{2}(\bmod p)$, Pacific J. Math., 5 (1955), 103-118.

Received March 3, 1982 and in revised form June 7, 1982. Supported by NSF grant MCS 81-01860.

University of California, San Diego

LA JOLla, CA 92093 


\section{PACIFIC JOURNAL OF MATHEMATICS \\ EDITORS}

DONALD BABBITT (Managing Editor)

University of California

Los Angeles, CA 90024

Hugo RossI

University of Utah

Salt Lake City, UT 84112

C. C. MOOre and ARThur OGus

University of California

Berkeley, CA 94720
J. Dugundi

Department of Mathematics

University of Southern California

Los Angeles, CA 90089-1113

R. FINN and H. SAMELSON

Stanford University

Stanford, CA 94305

ASSOCIATE EDITORS

$\begin{array}{lllll}\text { R. Arens } & \text { E. F. Beckenbach } & \text { B. H. Neumann } & \text { F. Wolf } & \text { K. Yoshida }\end{array}$ $(1906-1982)$

\section{SUPPORTING INSTITUTIONS}

UNIVERSITY OF ARIZONA

UNIVERSITY OF BRITISH COLUMBIA

CALIFORNIA INSTITUTE OF TECHNOLOGY

UNIVERSITY OF CALIFORNIA

MONTANA STATE UNIVERSITY

UNIVERSITY OF NEVADA, RENO

NEW MEXICO STATE UNIVERSITY

OREGON STATE UNIVERSITY
UNIVERSITY OF OREGON

UNIVERSITY OF SOUTHERN CALIFORNIA

STANFORD UNIVERSITY

UNIVERSITY OF HAWAII

UNIVERSITY OF TOKYO

UNIVERSITY OF UTAH

WASHINGTON STATE UNIVERSITY

UNIVERSITY OF WASHINGTON 


\section{Pacific Journal of Mathematics}

\section{Vol. 110, No. $1 \quad$ September, 1984}

Wojciech Abramczuk, A class of surjective convolution operators $\ldots \ldots \ldots \ldots 1$

K. Adachi, Extending bounded holomorphic functions from certain

subvarieties of a weakly pseudoconvex domain $\ldots \ldots \ldots \ldots \ldots \ldots$

Malvina Florica Baica, An algorithm in a complex field and its application

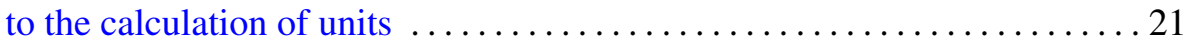

Giuliana Bianchi and Robert Cori, Colorings of hypermaps and a

conjecture of Brenner and Lyndon $\ldots \ldots \ldots \ldots \ldots \ldots \ldots \ldots \ldots \ldots \ldots \ldots$

Ronald James Evans, Determinations of Jacobsthal sums . . .......... 49

Leslie Foged, Characterizations of $\aleph$-spaces .................... 59

Nassif A. Ghoussoub and Paulette Saab, Weak compactness in spaces of

Bochner integrable functions and the Radon-Nikodým property . . . . . . 65

J. Gómez Gil, On local convexity of bounded weak topologies on Banach

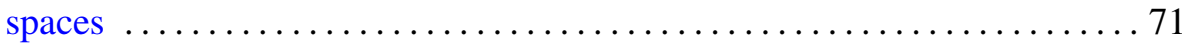

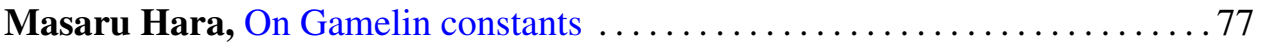

Wilfried Hauenschild, Eberhard Kaniuth and Ajay Kumar, Harmonic

analysis on central hypergroups and induced representations $\ldots \ldots \ldots 83$

Eugenio Hernandez, An interpolation theorem for analytic families of

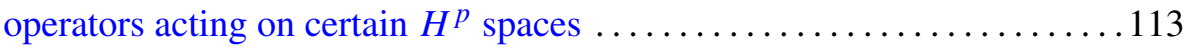

Thomas Alan Keagy, On "Tauberian theorems via block-dominated

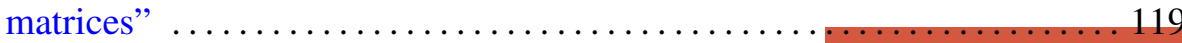

Thomas Landes, Permanence properties of normal structure $\ldots \ldots \ldots \ldots \ldots 125$

Daniel Henry Luecking, Closed ranged restriction operators on weighted

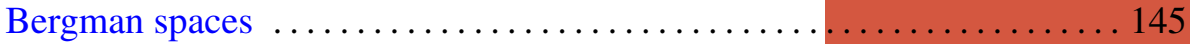

Albert Thomas Lundell, The $p$-equivalence of $\mathrm{SO}(2 n+1)$ and $\mathrm{Sp}(n) \ldots \ldots 161$

Mark D. Meyerson, Remarks on Fenn's "the table theorem" and Zaks' "the chair theorem" ..................................... 167

Marvin Victor Mielke, Homotopically trivial toposes . . . . . . . . . . 171

Gerard J. Murphy, Hyperinvariant subspaces and the topology on Lat A . . 183

Subhashis Nag, On the holomorphy of maps from a complex to a real manifold

Edgar Milan Palmer and Robert William Robinson, Enumeration of self-dual configurations ................................. 203

John J. Walsh and David Clifford Wilson, Continuous decompositions

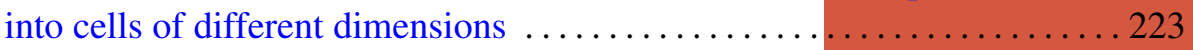

Walter John Whiteley, Infinitesimal motions of a bipartite framework .....233 